\title{
CATION-EXCHANGE SOLID-PHASE AND LIQUID-LIQUID EXTRACTION FOR THE DETERMINATION OF KHAT ALKALOIDS BY REVERSED PHASE HPLC-DAD
}

\author{
Minaleshewa Atlabachew ${ }^{1}$, Sandra Combrinck ${ }^{2 *}$ and Rob I. McCrindle ${ }^{1}$ \\ ${ }^{1}$ Department of Chemistry and ${ }^{2}$ Department of Pharmaceutical Sciences, Tshwane University of \\ Technology, Private Bag X680, Pretoria 0001, South Africa
}

(Received February 11, 2015; revised May 21, 2015)

\begin{abstract}
Leaves of khat (Catha edulis) are masticated to elicit their psycho-stimulating properties, resulting from the presence of the phenylpropylamino alkaloids. The determination of these alkaloids is important in pharmacological, phytochemical, forensic and law enforcement environments. In this study, the use of strong cation exchange-solid phase extraction (SCX-SPE) was investigated as an alternative means of sample purification prior to the determination of cathinone, cathine and norephedrine by reversed phase $\left(\mathrm{C}_{18}\right)$ high performance liquid chromatography (HPLC). Extraction parameters for SCX, including loading capacity and washing solvents, were established. An existing liquid-liquid extraction (LLE) method was improved in terms of recoveries obtained, by using ethyl acetate as extractant. For pure standards of the khat alkaloids, recoveries ranged from 83 to $97 \%$. Preconcentration, using a Genevac evaporator after the addition of acidified water to the sample, restricted analyte losses when compared to concentration under nitrogen. Although comparable recoveries were obtained when preconcentration was achieved in the presence of acidified water by rotary evaporation, this method is not suitable for large sample numbers. Best recoveries, ranging from 94 to $102 \%$, were obtained by SCX from spiked samples. Although the extraction efficiencies of LLE were lower (87-90\%), LLE yielded less complex chromatograms, indicating a purer extract.
\end{abstract}

KEY WORDS: Khat alkaloids, Solid phase extraction, Liquid-liquid extraction, HPLC, Genevac

\section{INTRODUCTION}

Khat (Catha edulis Vahl. Endl.) is an evergreen shrub or tree belonging to the Celastraceae family. Although the plant originates from Ethiopia, it occurs in Kenya, Malawi, Uganda, Tanzania, the Arabian Peninsula, Zambia, Zimbabwe and South Africa [1, 2]. The leaves are valued by millions of people, mainly in Africa and the Middle East, for its psychostimulatory effects. Khat has been declared illegal in many countries including 15 EU member states, Norway [3], the USA [4] and Australia [5]. The illegal use of khat in these countries is largely confined to ethnic communities and immigrants accustomed to prolonged chewing of the young leaves [6-8].

The phenylpropylamino alkaloids: $(-)$-cathinone $[(S)-\alpha$-aminopropiophenone $],(+)$-cathine $[(1 S)(2 S)$-norpseudoephedrine] and (-)-norephedrine [(1R) $(2 S)$-norephedrine] [9] are the main psychostimulant compounds present in khat and are considered to be the addictive agents [10]. In addition to these, about 200 compounds, including volatile organic compounds, sterols and triterpenes, more than 10 amino acids, ascorbic acid, flavonoids, tannins (7-14\% by weight in dried leaves) and about 62 cathedulins have been identified in khat $[7,11]$.

Environmental and climatic conditions, as well as local traditions practiced with regard to cultivation and harvesting, determine the general appearance and chemical profile of khat leaves [12]. Large variations in the alkaloid content of khat leaves have been reported. The phenyalkylamine fraction of young shoots was found to comprise more than $90 \%(-)$-cathinone $[13,14]$, which has a more powerful psychostimulant effect than cathine and norephedrine [15]. During maturation, cathinone is enzymatically converted to cathine and (-)-norephedrine [9]. Sunlight- and heat-induced degradation of cathinone to cathine and norephedrine also takes place during drying of the leaves or extraction of cathinone in the laboratory $[13,16]$. The

*Corresponding author. E-mail: combrincks@tut.ac.za 
instability of cathinone is, at least in part, to blame for the variable alkaloid content of khat reported in the literature.

Several analytical methods involving gas chromatography-mass spectrometry (GC-MS) [9, 17, 18], gas chromatography-flame ionization detection (GC-FID) [8], high performance liquid chromatography-diode array detection (HPLC-DAD) [19-22], liquid chromatography-tandem mass spectrometry (LC-MS/MS) [23] and capillary electrophoresis-diode array detection (ECDAD) [24] have been developed for the determination of the phenylpropylamino alkaloids in khat leaf samples. These methods have been applied in pharmacological, phytochemical, forensic and law enforcement environments.

The complexity of the leaf matrix dictates that analysis is preceded by some form of sample clean-up. Sample preparation is aimed at ensuring reliable and repeatable results by enriching the analytes, while reducing matrix effects in subsequent chromatographic analyses. Typical acid-base alkaloid extraction has been widely reported for the purification of khat leaf extracts destined for GC analysis. After liquid-liquid extraction (LLE), the organic solvent containing the alkaloids is removed under a gentle stream of nitrogen or under reduced pressure on a rotary evaporator and analysed directly [8, 9, 18, 25-27]. Geisshusler and Brenneisen [19] proposed a lengthy LLE procedure, followed by precipitation of the alkaloids with oxalic acid, prior to their determination by normal phase HPLC-DAD. It can be reasonably assumed that the introduction of a precipitation step may result in the loss of analytes, thereby affecting the accuracy and reproducibility of the method.

Atlabachew et al. [22] reported the use of matrix solid-phase dispersion for extraction and clean-up of the alkaloids from khat leaves, prior to HPLC-DAD detection. Despite the procedure being simple and rapid, it was found to be ineffective for eliminating co-extractives.

Solid-phase extraction (SPE) has emerged as a powerful tool for the purification and enrichment of a range of organic compounds from various environmental and biological matrices. Mathys and Brenneisen [20] applied SPE with a $\mathrm{C}_{18}$ sorbent, for the purification of khat extracts prior to reversed phase-HPLC analysis. Briefly, the khat sample was extracted by ultrasonication and the combined acidified aqueous extracts were evaporated to dryness at 65 ${ }^{\circ} \mathrm{C}$. The dried extract was then dissolved in $20 \%$ aqueous acetonitrile, containing $8.5 \mathrm{~g} / \mathrm{L}$ orthophosphoric acid and $200 \mu \mathrm{L} / \mathrm{L}$ hexylamine, and applied to a pre-conditioned cartridge. This was then eluted with the same solvent, in the absence of a washing step, which may result in high concentrations of contaminants. Furthermore, preconcentration of the aqueous extract is time consuming and the temperature required for solvent evaporation may affect the stability of cathinone. Cation-exchange SPE is a selective technique applied for the extraction of basic compounds, amongst others, from various matrices. The sorbent is used to retain or release analytes by appropriately adjusting the prevailing $\mathrm{pH}$ [28]. In spite of the ease with which the phenyalkylamine alkaloids can be protonated or deprotonated, the use of strong cation exchange (SCX)-SPE for their partial purification from khat leaves has not yet been reported.

In this study, the addition of acidified water as part of the preconcentration process was investigated, with the aim of stabilising cathinone throughout the extraction. A SCX-SPE cleanup method was developed and validated for the determination of khat alkaloids from leaf extracts. Furthermore, the LLE method of Geisshusler and Brenneisen [18] was modified by using an alternative extractant to improve efficiencies.

\section{EXPERIMENTAL}

\section{Preparation of standards}

(-)-Norephedrine [(1R,2S)-norephedrine], (-)-cathine hydrochloride and (+)-ephedrine hydrochloride (internal standard; IS) were purchased from Sigma Aldrich (Aston Manor, South Africa). Cathinone was isolated as the oxalate salt from khat leaves and the purity was found to 
be in excess of $90 \%$ [13]. Stock solutions, with a concentration of $1.00 \mathrm{mg} / \mathrm{mL}$ were prepared from each of the standards in deionised ultrapure water (Econopure, GIC Scientific, Florida, South Africa; $>18.2 \mathrm{M} \Omega \mathrm{cm})$, acidified with phosphoric acid $(\mathrm{pH}=1.76)$. Eight calibration standards over the range $0.500-200 \mu \mathrm{g} / \mathrm{mL}$ were prepared in acidified water.

\section{Sample preparation}

Two khat samples were collected from the Soutpansberg region, Limpopo, South Africa, and immediately frozen to prevent decomposition of cathinone $\left(-18^{\circ} \mathrm{C}\right)$. The young leaves (normally chewed) were freeze-dried and then ground with a mortar and pestle. Several samples for subsequent analysis were prepared by accurately measuring $0.25 \mathrm{~g}$ portions of the first sample (Sample A), mixed with $500 \mu \mathrm{L}$ of $1.00 \mathrm{mg} / \mathrm{mL}$ ephedrine hydrochloride (IS), and extracting twice with $10.00 \mathrm{~mL}$ and once with $5.00 \mathrm{~mL}$ of $2 \%$ aqueous formic acid (AAR grade, SMM Instruments) in an ultrasonic bath (Bransonic Ultrasonics Corporation, Mexico) for a total of 60 min (extract from Sample A). The extracts were characterised by a reddish colour. Each extract prepared from Sample A $(0.25 \mathrm{~g})$, containing $500 \mu \mathrm{g}$ of IS, was then spiked with 100.0, 100.0 and $150.0 \mu \mathrm{g}$ of cathinone, cathine and norephedrine, respectively. In parallel, a second set of extracts was prepared from Sample A as described, but these were not spiked. The second sample (Sample B) was treated in the same way, without the addition of internal standard or analytes.

\section{Solid-phase extraction}

Development of the protocol. An Accubond SCX cartridge $(500 \mathrm{mg}, 6 \mathrm{~mL}$; J\&W Scientific, California, USA) was conditioned with $2 \times 4 \mathrm{~mL}$ of methanol (HPLC grade; LAB-SCAN Analytical Sciences, Gliwice, Poland), followed by equilibration with $4 \mathrm{~mL}$ of deionised water and then $4 \mathrm{~mL}$ of $2 \%$ aqueous formic acid. The conditioned cartridges were loaded with 5.00 $\mathrm{mL}$ of extract from Sample B in $2 \%$ formic acid. This was followed by the washing steps, performed sequentially with $2 \times 4.0 \mathrm{~mL}$ of $0.2 \%$ formic acid and $2 \times 4.0 \mathrm{~mL}$ methanol. After drying the cartridge for $30 \mathrm{~s}$ under vacuum, the analytes were eluted with $8.0 \mathrm{~mL}$ of $0.10,0.50$, $0.75,1.00,2.00$ or $4.00 \% \mathrm{NH}_{4} \mathrm{OH}$ (AAR grade, SMM Instruments) in methanol. All the steps were conducted at a flow rate of 1 to $2 \mathrm{~mL} \mathrm{~min}^{-1}$ using a manifold (Waters; Microsep, Johannesburg, South Africa) connected to a vacuum pump (Vacuubrand, GMBH, Germany). The eluates were dried under a gentle stream of nitrogen using a Reacti-vap evaporating unit (Model 18780; Thermo Scientific, Randburg, South Africa) at room temperature. After removal of about $6 \mathrm{~mL}$ of the solvent, $2 \mathrm{~mL}$ of acidified water $(\mathrm{pH}=1.76)$ was added and evaporation continued until the final volume was reduced to near dryness. The concentrate was quantitatively transferred and the volume was adjusted to $2.00 \mathrm{~mL}$. Eluents were filtered through Acrodisc syringe filters (PVDF membrane; $0.45 \mu \mathrm{m}$ pore size; Merck, South Africa) and analysed by HPLC-DAD. A concentration of $2.00 \% \mathrm{NH}_{4} \mathrm{OH}$ was used as eluent in all subsequent purifications.

Loading capacity of the cartridge. The loading capacity of the SCX cartridge was established before carrying out the quantitative studies. Four volumes of unspiked extract from Sample A $(2.50,5.00,7.50$ and $10.00 \mathrm{~mL})$ were applied to individual preconditioned cartridges, which were washed as described. Elution was achieved using $2.00 \%$ methanolic $\mathrm{NH}_{4} \mathrm{OH}$. The eluates were evaporated as described and reconstituted with acidified water $(\mathrm{pH} 1.76)$ prior to HPLCDAD analysis. No trace of any of the analytes was observed in the washing discards, proving that no breakthrough of analytes took place at these concentrations or loading volumes. Finally, a sample volume of $5.00 \mathrm{~mL}$ was selected for future loading, since this would compensate for 
potentially higher alkaloid concentrations in samples, while ensuring that trace levels of analytes, in particular norephedrine, could be detected.

Reproducibility and recoveries. Using the SCX-SPE method, followed by HPLC-DAD analysis, the concentrations of cathinone, cathine and norephedrine in unspiked Sample A were determined as $25.8,27.6$ and $1.44 \mu \mathrm{g} / \mathrm{mL}$, respectively. Portions $(5.00 \mathrm{~mL})$ of the spiked extract prepared from Sample A, containing $500 \mu \mathrm{g}$ of IS, was extracted using the SCX-SPE procedure. Three replicates were included for each extraction and two replicate HPLC-DAD analyses were performed on each filtrate. The unspiked sample was analysed in parallel. The recovery of each alkaloid was expressed as the difference in the concentrations of the spiked and unspiked samples as a percentage of the concentration spiked.

\section{Liquid-liquid extraction (LLE)}

Development of the protocol. The LLE extraction procedure of Geisshusler and Brenneisen [19] was optimised in terms of the organic extraction solvent and the method for removal of the solvent prior to HPLC analysis. To select the organic solvent, acidified water $(\mathrm{pH}=1.76)$ was supplemented with the three alkaloid standards to a final concentration of $20.0 \mathrm{mg} / \mathrm{L}$ each. A $5.00 \mathrm{~mL}$ aliquot (in triplicate) was basified to $\mathrm{pH} 9-10$ with $10 \% \mathrm{NaOH}$ solution and extracted with $3 \times 5.00 \mathrm{~mL}$ of diethyl ether or ethyl acetate (AAR grade, SMM instruments). The individual organic phases were subsequently mixed with $2 \mathrm{~mL}$ of the acidified water. After concentration under a gentle stream of nitrogen at room temperature, the volume of each was adjusted to $2.00 \mathrm{~mL}$ and analysed by HPLC.

For optimisation of the drying procedure, a portion of the extract prepared from Sample B $(5.00 \mathrm{~mL})$ was transferred to a $15 \mathrm{~mL}$ nylon centrifuge tube and extracted twice with a $5 \mathrm{~mL}$ portion of ethyl acetate, which was subsequently discarded. Sufficient aqueous $\mathrm{NaOH}$ was added to render the aqueous solution basic $(\mathrm{pH} 9-10)$, where after the solution was extracted with ethyl acetate as described. The combined extracts were evaporated under different conditions.

Rotary evaporation (Buchi rotavapor R-200, Labotec, South Africa): The ethyl acetate extracts were dried at $30^{\circ} \mathrm{C}$ under reduced pressure and the residues were reconstituted with 2.0 $\mathrm{mL}$ of water, acidified with phosphoric acid, $\mathrm{pH}=1.76$. A second set of ethyl acetate extracts was treated by mixing each extract with $2.0 \mathrm{~mL}$ of acidified water before concentrating at $30{ }^{\circ} \mathrm{C}$ under reduced pressure. The solution was quantitatively transferred to an eppendorf tube and the volume was adjusted to $2.0 \mathrm{~mL}$ with acidified water.

Genevac evaporator (EZ 2.3, SP Scientific, New York, USA): The ethyl acetate extracts were vacuum dried in the Genevac evaporator (temperature maximum $30{ }^{\circ} \mathrm{C}$ ) until dryness and the residues were then reconstituted to a final volume of $2.0 \mathrm{~mL}$ with acidified water. A second set of ethyl acetate extracts were mixed with $2.0 \mathrm{~mL}$ of the acidified water, placed in the Genevac and the solvent evaporated for approximately $1 \mathrm{~h}$ until less than $2.0 \mathrm{~mL}$ of the aqueous phase remained. Finally the volume was adjusted to $2.0 \mathrm{~mL}$ with acidified water.

Evaporation under nitrogen: The extracts were dried under a gentle stream of nitrogen at room temperature and then reconstituted to $2.0 \mathrm{~mL}$ with acidified water.

Recoveries of khat alkaloids from LLE. It was found that cathinone was stabilised by the addition of the acidified water prior to concentration. This procedure for drying was therefore applied for the recovery study. A $5.00 \mathrm{~mL}$ portion of the spiked extract from Sample A, containing $500 \mu \mathrm{g}$ of ephedrine hydrochloride (IS), was extracted in triplicate with ethyl acetate as described. The ethyl acetate extract was mixed with $2.0 \mathrm{~mL}$ of acidified water and placed in the Genevac evaporator. Once the organic phase was removed, the remainder $(\approx 1.5 \mathrm{~mL})$ was adjusted to $2.0 \mathrm{~mL}$ with the acidified water, filtered and analysed by HPLC. An unspiked 
sample was analysed in parallel. The recovery of each alkaloid was expressed as the difference in the concentrations of the spiked and unspiked samples as a percentage of the concentration spiked.

\section{HPLC analysis}

The analyses were performed using an Agilent 1200 Series HPLC (Agilent Technologies Inc., Chemetrix, South Africa), equipped with a binary pump and fitted with an Ascentis ${ }^{\mathrm{TM}} \mathrm{C}_{18}$ column (Supelco; $25 \mathrm{~cm}$ x $4.6 \mathrm{~mm}$ × $5 \mu \mathrm{m}$ particle size). A $5.00 \mu \mathrm{L}$ sample volume was analysed throughout. The diode array detector was used for quantification at $200 \mathrm{~nm}$. The mobile phase consisted of $85 \%$ aqueous phosphoric acid (Solvent $\mathrm{A} ; \mathrm{pH} 1.76$ ) and $10 \%$ aqueous acetonitrile (Solvent B, Lab-scan, Analytical Science, Poland) at a flow rate of $1.5 \mathrm{~mL} / \mathrm{min}$. A linear gradient was applied from 0 to $70 \%$ Solvent B in 20 min. Internal standard calibration curves were constructed over the range 0.50 to $200 \mu \mathrm{g} / \mathrm{mL}$, by analysing the pure calibration standards. The limits of detection (LOD) and quantification (LOQ) were calculated using the formula $L O D=3 \mathrm{~S}_{\mathrm{a}} / \mathrm{b}$ and $\mathrm{LOQ}=10 \mathrm{~S}_{\mathrm{a}} / \mathrm{b}$, where $\mathrm{S}_{\mathrm{a}}$ is the uncertainty of the intercept and $\mathrm{b}$ is the sensitivity of the method [29].

\section{Statistical analysis}

Paired t-test and one-way ANOVA using IBM SPSS Statistics 20 were used to verify whether there was a significant difference in the data obtained from different solvents/methods and sample preconcentration methods, respectively.

\section{RESULTS AND DISCUSSION}

\section{HPLC analysis}

Baseline resolution of the analytes and IS was achieved with a $\mathrm{C}_{18}$ column, without any amine modifiers in the mobile phase. Most liquid chromatography methods described are based on columns containing cation exchange or normal phase (Lichrosorb Si-60, p-Porasil) stationary phases. The use of reversed phase columns is common, but phenyl substituents, rather than aliphatic $\mathrm{C}_{8}$ or $\mathrm{C}_{18}$ stationary phases are used [19, 22, 23, 30]. Mathys and Brenneisen [20] and Chappell and Lee [21] made use of a $\mathrm{C}_{18}$ column, but added amine modifiers to the mobile phase. However, these modifiers are known to diminish the life span of the column, reduce the sensitivity of the detector and affect the baseline during gradient elution [31].

A linear detector response was obtained for each of the analytes as reflected by the regression values, which were above 0.999 over the concentration range 0.50 to $200 \mu \mathrm{g} / \mathrm{mL}$. The equations for the calibration curves were $y=0.042 x+0.0 .024\left(R^{2}=0.9999\right)$ for cathinone, $\mathrm{y}=0.022 \mathrm{x}+0.019\left(\mathrm{R}^{2}=0.9997\right)$ for cathine and $\mathrm{y}=0.020 \mathrm{x}+0.015\left(\mathrm{R}^{2}=0.9997\right)$ for norephedrine. The greater value of the $\mathrm{x}$ variable for cathinone indicates a substantially higher sensitivity of the detector towards this compound. The LOD values obtained for cathinone, cathine and norephedrine were $0.91 ; 1.50$ and $1.72 \mu \mathrm{g} / \mathrm{mL}$, respectively. The corresponding LOQ values were $3.03,5.00$ and $5.73 \mu \mathrm{g} / \mathrm{mL}$, respectively.

\section{SCX-SPE clean-up}

The SPE protocol used was adapted from methods previously reported for the purification of basic drugs and technical notes of the manufacturer [32, 33]. In acidic media, the three alkaloids exist in their protonated form, making cation exchange a logical choice for sample purification. The strong interactions between the analytes and the resin allowed contaminants to be removed 
during the washing steps, without significant sample loss. Methanolic $\mathrm{NH}_{4} \mathrm{OH}$, used as eluent, served to break the ionic interactions between the analytes and the sorbent, while the methanol prevented adsorption interactions. Six concentrations of $\mathrm{NH}_{4} \mathrm{OH}$ were tested (Table 1). The lowest yields of all the alkaloids were obtained when using $0.10 \% \mathrm{NH}_{4} \mathrm{OH}$ as eluent. Thereafter, the yield increased until a plateau was reached, corresponding to complete desorption of the analytes from the cation-exchange resin. It was therefore decided to apply $2 \% \mathrm{NH}_{4} \mathrm{OH}$ in $\mathrm{MeOH}$ as elution solvent, since the use of higher concentrations affected the sharpness of the peaks obtained by HPLC analysis. Khat extracts contain large amounts of anthocyanins, evident from the red colour of the extract. Unfortunately the anthocyanins are also protonated in acidic medium and interact strongly with the SCX adsorbent.

Table 1. Concentrations (mean $\pm \mathrm{SD} \mu \mathrm{g} / \mathrm{mL}$ ) of khat alkaloids recovered from SCX-SPE cartridges as determined by HPLC-DAD to establish the optimal $\mathrm{NH}_{4} \mathrm{OH}$ required for desorption from the cartridge after loading of Sample B.

\begin{tabular}{|c|c|c|c|}
\hline \multirow{2}{*}{$\% \mathrm{NH}_{4} \mathrm{OH}$} & \multicolumn{3}{|c|}{ Concentration $(\mu \mathrm{g} / \mathrm{mL})$} \\
\cline { 2 - 4 } & Cathinone & Cathine & Norephedrine \\
\hline 0.10 & $80.8 \pm 6.5$ & $13.1 \pm 0.9$ & $2.1 \pm 0.1$ \\
\hline 0.50 & $95.9 \pm 5.8$ & $14.9 \pm 1.0$ & $2.7 \pm 0.2$ \\
\hline 0.75 & $100 \pm 4$ & $18.4 \pm 0.8$ & $2.8 \pm 0.1$ \\
\hline 1.00 & $103 \pm 4$ & $17.7 \pm 0.6$ & $3.3 \pm 0.1$ \\
\hline 2.00 & $106 \pm 4$ & $18.5 \pm 1.2$ & $3.0 \pm 0.2$ \\
\hline 4.00 & $103 \pm 3$ & $18.1 \pm 0.7$ & $3.1 \pm 0.2$ \\
\hline
\end{tabular}

Although a $5.00 \mathrm{~mL}$ sample loading volume was selected for SCX clean-up, volumes of up to $10.00 \mathrm{~mL}$ may be applied for samples containing low concentrations of the alkaloids, since no analyte breakthrough from the resin was obtained for the larger volumes. The percentage recoveries obtained were $94.1 \pm 10.4,104 \pm 11$ and $95.4 \pm 8.5 \%$ for cathinone, cathine and norephedrine, respectively $(n=6)$, indicating acceptable reproducibility. The chromatograms of the SCX eluents from unspiked and spiked khat extract, together with that of the standards, are provided in Figure 1.

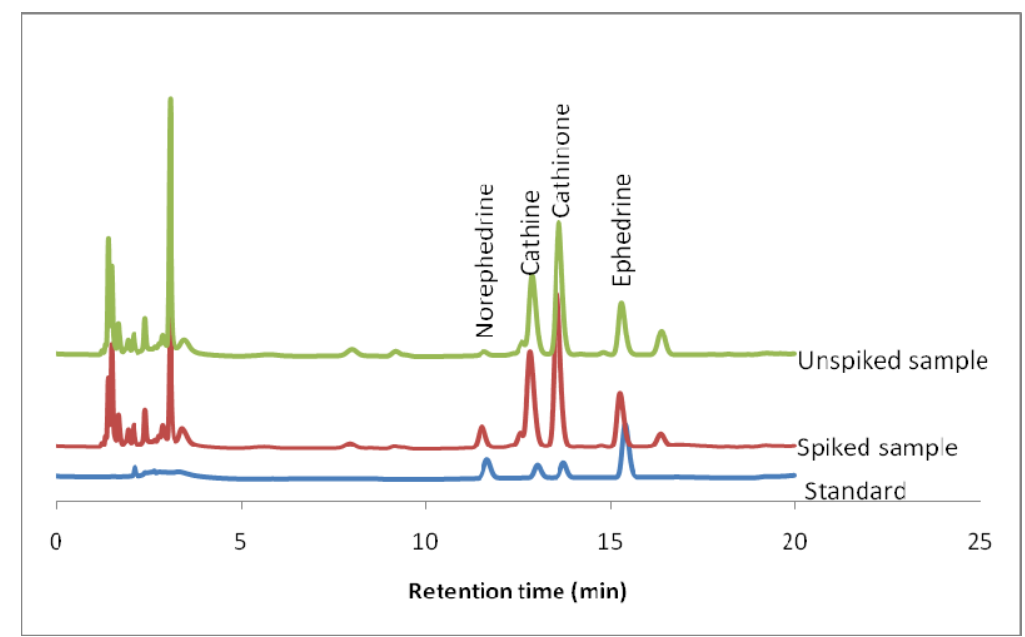

Figure 1. Chromatograms of unspiked and spiked khat samples A, following purification with SCX-SPE under optimized conditions, together with standards using HPLC-DAD. Values on the vertical axis are the same for all three chromatograms.

Bull. Chem. Soc. Ethiop. 2015, 29(3) 


\section{Evaluation of effect of solvent evaporation techniques from LLE protocol}

Diethyl ether is commonly used to isolate khat alkaloids from the aqueous extract, since the low boiling point facilitates evaporation at low temperatures $[9,14,18,19,22]$. When ethyl acetate was used as extractant, the recoveries of all three alkaloids were higher (Table 2). However, the recoveries of cathinone and norephedrine were found to be significantly different $(p<0.05)$. Extraction by the first $5.00 \mathrm{~mL}$ volume resulted in an increase of 52, 79 and $100 \%$ of cathinone, cathine and norephedrine, respectively, when ethyl acetate was used, rather than diethyl ether. This indicates a more efficient removal of the alkaloids from the aqueous layer by ethyl acetate. If diethyl ether is to be used, more than three extractions are recommended. Although diethyl ether is easily removed, the high volatility of the solvent complicates the extraction. Consequently, ethyl acetate was found to be an appropriate solvent for quantitative recovery of the alkaloids and was selected for the present study.

Table 2. Percentage recoveries (mean $\pm \mathrm{SD}$ ) for LLE of khat alkaloids by diethyl ether or ethyl acetate.

\begin{tabular}{|c|c|c|c|c|c|c|}
\hline \multirow{3}{*}{ Extraction } & \multicolumn{6}{|c|}{ Percentage extracted (mean \pm standard deviation) } \\
\cline { 2 - 7 } & \multicolumn{3}{|c|}{ Diethyl ether } & \multicolumn{3}{c|}{ Ethyl acetate } \\
\cline { 2 - 7 } & Cathinone & Cathine & Norephedrine & Cathinone & Cathine & Norephedrine \\
\hline 1 & $54.2 \pm 4.6$ & $35.5 \pm 2.7$ & $28.5 \pm 2.7$ & $82.4 \pm 4.6$ & $63.6 \pm 2.8$ & $56.9 \pm 2.0$ \\
\hline 2 & $23.6 \pm 1.4$ & $27.2 \pm 1.8$ & $23.8 \pm 2.0$ & $13.9 \pm 0.1$ & $15.3 \pm 1.2$ & $17.8 \pm 1.4$ \\
\hline 3 & $8.7 \pm 0.8$ & $17.6 \pm 2.7$ & $18.7 \pm 2.7$ & $1.0 \pm 0.1$ & $7.84 \pm 1.6$ & $8.2 \pm 1.1$ \\
\hline Total & $86.6 \pm 4.8$ & $80.3 \pm 4.2$ & $71.0 \pm 4.3$ & $97.3 \pm 4.6$ & $86.8 \pm 3.4$ & $82.9 \pm 2.7$ \\
\hline
\end{tabular}

The evaporation of the solvent from the extracts was identified as a potential source of analyte losses. Complete drying accelerates the decomposition and diamerization of cathinone [16]. Since ethyl acetate is unsuitable for sample dilution in reversed phase HPLC analysis, the organic solvent was removed prior to injection. The effects of rotary evaporation and the use of the Genevac evaporator, with and without the addition of acidified water, as well as evaporation with a stream of nitrogen, were compared.

Although complete evaporation of ethyl acetate in the Genevac evaporator significantly reduced the yields of all three analytes $(\mathrm{p}<0.05)$, the addition of acidified water to extracts evaporated in the Genevac and on the rotary evaporator yielded the highest recoveries (Table 3 ). Cathinone is unstable at elevated temperatures, particularly when present in alkaline solutions or in the solid state [16]. However, the compound is fairly stable in acidic media and hence the addition of acidified water. In addition, Genevac vacuum drying is appropriate for large sample numbers, since 40 samples can be simultaneously concentrated or dried and no manual intervention is required.

Higher yields were obtained when complete drying was done by rotary evaporation (Table 3 ), instead of by Genevac, probably because the time interval between evaporation and reconstitution with mobile phase was short. Cathinone was fairly stable in ethyl acetate, even at $30{ }^{\circ} \mathrm{C}$ for more than $1 \mathrm{~h}$. Once dried, however, the solid rapidly decomposes. Drying under rotary evaporation in the absence of acidified water reduced the yields of the khat alkaloids. The best yield was obtained for cathinone (Table 3) when drying was done in the presence of acidified water by rotary evaporation. This result confirms the stabilising effect of the acidic mobile phase on cathinone and restricts losses on the walls of the evaporation flask.

Although acceptable recoveries were obtained after drying under a gentle stream of nitrogen at room temperature, it is difficult to handle many samples simultaneously. The flow rate of the nitrogen must be meticulously controlled to prevent loss of the analytes. Based on practical considerations and high recoveries obtained, it was decided to concentrate samples in the Genevac, but in the presence of acidified water. 
Table 3. Concentrations (mean $\pm \mathrm{SD} \mu \mathrm{g} / \mathrm{mL}, \mathrm{n}=6$ ) of khat alkaloids recovered from Sample B following LLE and various methods of preconcentration. The same uppercase letter indicates that values do not differ significantly $(\mathrm{p}>0.05)^{*}$, while different uppercase letters indicate significant differences.

\begin{tabular}{|c|c|c|c|c|}
\hline Preconcentration method & Cathir & & Cathine & Norephedrine \\
\hline Rotavapor & $77.1 \pm 1.5$ & $\mathrm{~A}$ & $12.5 \pm 0.7 \quad \mathrm{E}$ & $3.08 \pm 0.28 \quad G$ \\
\hline M. phase + rotavapor & $104 \pm 6$ & $\mathrm{~B}$ & $14.6 \pm 1.0 \quad \mathrm{~F}$ & $3.62 \pm 0.27 \quad \mathrm{H}$ \\
\hline Genevac & $26.9 \pm 0.5$ & $\mathrm{C}$ & $11.2 \pm 0.5 \quad \mathrm{E}$ & $2.33 \pm 0.29 \quad \mathrm{I}$ \\
\hline M. phase + Genevac & $98.7 \pm 1.9$ & $\mathrm{~B}$ & $14.8 \pm 1.1 \quad \mathrm{~F}$ & $3.39 \pm 0.22 \mathrm{GH}$ \\
\hline Stream of nitrogen & $87.0 \pm 0.6$ & $\mathrm{D}$ & $12.7 \pm 1.2 \mathrm{EF}$ & $2.95 \pm 0.19 \quad \mathrm{G}$ \\
\hline
\end{tabular}

*One way ANOVA using IBM SPSS Statistics 20 was used.

Comparison of SCX with LLE

The yields obtained by SCX extraction were compared with those from LLE; extracts were preconcentrated in the Genevac vacuum evaporator in the presence of acidified water. Higher yields were obtained using SCX-SPE than with LLE $(94.1 \pm 10.4,104 \pm 11$ and $95.4 \pm 8.5 \%$ versus $90.1 \pm 9.2 ; 88.9 \pm 8.2$ and $87.5 \pm 6.0 \%$ for cathinone, cathine and norephedrine respectively). Although similar yields ( $p>0.05$ for paired t-test) were obtained from both methods, LLE resulted in cleaner extracts, but is more laborious than SCX, particularly when several samples are analysed simultaneously.

\section{CONCLUSIONS}

The study was aimed at improving pre-analysis purification of khat leaf samples by using SCX, rather than reversed phase SPE cartridges, prior to the determination of the phenyalkylamine alkaloids. An appropriate elution solvent and loading capacity were determined. The existing LLE method was modified by the replacement of diethylether with ethyl acetate to improve extraction efficiency. It is recommended that SCX-SPE be used for purification, together with Genevac evaporation, particularly when large sample numbers are involved. Cartridge extraction using a vacuum manifold requires less manual intervention than LLE.

\section{ACKNOWLEDGMENTS}

The authors would like to thank Tshwane University of Technology (TUT) for financial support. Minaleshewa Atlabachew is thankful to Bahir Dar University for the provision of leave of absence during his visit to TUT.

\section{REFERENCES}

1. Field, C.R. Alkaloids of Catha spp., MSc. Dissertation, University of Natal: Pietermaritzburg; South Africa; 2001; p 100.

2. Al-Motarreb, A.; Baker, K.; Broadley, K.J. Phytother. Res. 2002, 16, 403.

3. EMCDDA (European Monitoring Centre for Drug and Drug Addiction). Available at: http://www.drugabuse.gov/publications. Accessed in November, 2014.

4. NIDA (National Institute for Drug Abuse). Available at: http://www.drugabuse.gov/publications. Accessed in November, 2014.

5. AGDH (Australian Government Department of Health). Available at: http://www.health.gov.au. Accessed in November, 2014.

6. Odenwald, M. Sucht. 2007, 53, 9. 
Cation-exchange solid-phase and liquid-liquid extraction for determination of khat alkaloids 339

7. Feyissa, A.M.; Kelly, J.P. Biol. Psych. 2008, 32, 1147.

8. Gambaro, V.; Arnoldi, S.; Colombo, M.L.; Dell'Acqua, L.; Guerrini, K.; Roda, G. Forensic Sci. Int. 2012, 217, 87.

9. Krizevski, R.; Dudai, N.; Bar, E.; Lewinsohn, E. J. Ethnopharmacol. 2007, 114, 432.

10. Brenneisen, R.; Fisch, H.U.; Koelbing, U.; Geisshusler, S.; Kalix, P. Br. J. Clin. Pharm. 1990, 30, 825 .

11. Atlabachew, M.; Chandravanshi, B.S.; Redi, M. Int. J. Food Proper. 2014, 17, 45.

12. Nordal, A. Bull. Narc. 1980, 32, 51.

13. Atlabachew, M.; Chandravanshi, B.S.; Redi, M.; Torto, N.; Chigome, S.; Pule, B.O. Bull. Chem. Soc. Ethiop. 2013, 17, 347.

14. Dagne, E.; Adugna, Y.; Kebede, E.; Atilaw, Y. Ethiop. e-J. Res. Innov. Foresight 2010, 2 , 7.

15. Brenneisen, R.; Geisshusler, S.; Schorno, X. J. Pharm. Pharmacol. 1986, 38, 298.

16. Szendrei, K. Bull. Narc. 1980, 32, 5.

17. Ripani, L.; Schiavone S.; Garofano, L. Forensic Sci. Int. 1996, 78, 39.

18. Krizevski, R.; Dudai, N.; Bar, E.; Dessow, I.; Ravid, U.; Lewinsohn, E. Israel J. Plant Sci. 2008, 56, 207.

19. Geisshusler, S.; Brenneisen, R. J. Ethnopharmacol. 1987, 19, 269.

20. Mathys, K.; Brenneisen, R. Pharm. Acta Helv. 1993, 68, 121.

21. Chappell, J.S.; Lee, M.M. Forensic Sci. Int. 2010,195, 108.

22. Atlabachew, M.; Torto, N.; Chandravanshi, B.S.; Redi, M. Chromatographia 2013, 76, 401.

23. Dimba, E.A.O.; Gjertsen, B.T.; Bredholt, T.; Fossan, K.O.; Costea, D.E.; Francis, G.W.; Johannessen, A.C.; Vintermyr, O.K. Br. J. Cancer. 91, 2004, 91, 1726.

24. Roda, G.; Liberti, V.; Arnoldi, S.; Argo, A.; Rusconi, C.; Suardi, S.; Gambaro, V. Forensic Sci. Int. 2013, 228, 154.

25. Lebelle, M.J.; Lauriault, G.; Lavoie, A. Forensic Sci. Int. 1993, 61, 53.

26. Toennes, S.W.; Kauert, G.F. Clin. Chem. 2002, 48, 1715.

27. Toennes, S.W.; Harder, S.; Schramm, M.; Niess, C.; Kauert, G.F. Br. J. Clin. Pharmacol.2003, 56, 125.

28. Fontanals, N.; Miralles, N.; Abdullah, N.; Davies, A.; Gilart, N.; Cormack, P.A.G. J. Chromatogr. A 2014, 1343, 55.

29. Miller, J.C.; Miller, J.N. Statistics for Analytical Chemistry, 3rd ed., Ellis Horwood: Chichester; 1993; p 233.

30. El-Domiaty, M.M.; Elhag, H.M.; El-Feraly, F.S.; Al-Meshal, I.A.; El-Olemy, M.M. Int. J. Pharmacog. 1994, 32, 135.

31. Amersham Bioscience Reversed Phase Chromatography, Principles and Methods, AA ed., Uppsala: Sweden; 1999; p 86.

32. Logan, B.K.; Stafford, D.T. J. Anal. Toxicol. 1990, 14, 154.

33. Biotage; Isolute, Technical Note 106: Method development in solid phase extraction using ISOLUTE® SCX and SCX-3 SPE columns for the extraction of aqueous samples, 2004; 6. 